\title{
Expectation of Tourism Demand in Iraq by Using Artificial Neural Network
}

\author{
Rashid Anasari \\ Faculty of Humanities and Social Sciences, Department of Administration \& Economy, Koya University, \\ Koya, Iraq \\ ria_ans,soc11@koyauniversity.org
}

\begin{abstract}
This study survey and proves this effectiveness connected with artificial neural networks (ANNs) as an alternative approach in the tourism research. The learning utilizes the travel industry in the Japan being a method for estimating need to exhibit the solicitation. The outcome reveals the use of ANNs in tourism research might perhaps result in better quotations when it comes to prediction bias and accuracy. Even more applications of ANNs in the context of tourism demand examination is needed to establish and validate the effects. An ANN is based on a collection of connected units or nodes called artificial neurons, which loosely model the neurons in a biological brain. Each connection, like the synapses in a biological brain, can transmit a signal to other neurons. An artificial neuron that receives a signal then processes it and can signal neurons connected to it.
\end{abstract}

Keyword: Iraq; Tourism; Artificial Neural Network

\section{Introduction}

Considering tourist inspiration has consistently been a significant factor for the travel industry the board. The methods for communicating and the progressions that create in time in vacationer inspirations legitimately influence the travel industry request and supply. So as to make due in a market in consistent advancement and change, described by wild challenge, in an economy in emergency, providers of the travel industry items and administrations need to know and foresee changes in the inspirations that decide visitors to purchase an occasion bundle. Expansive point, vigorously discussed, visitor inspiration can be characterized as a mental upgrade ready to decide the longing to travel.

In ANN implementations, the "signal" at a connection is a real number, and the output of each neuron is computed by some non-linear function of the sum of its inputs. The connections are called edges. Neurons and edges typically have a weight that adjusts as learning proceeds. The weight increases or decreases the strength of the signal at a connection. Neurons may have a threshold such that a signal is sent only if the aggregate signal crosses that threshold. Typically, neurons are aggregated into layers. Different 
layers may perform different transformations on their inputs. Signals travel from the first layer (the input layer), to the last layer (the output layer), possibly after traversing the layer's multiple times.

Exact forecast and estimation of the travel industry request is significant really taking shape choice with respect to interests in both people in general and private parts, likewise in the transient advertising choices in the travel industries (Crouch, 1995; Lim, 1997; Song and Li, 2008; Witt and Witt, 1995). The travel industry request examination is significant and vital piece of the travel industry.

For instance, the movement business demand examination can give long and flashing data that are significant for any person who has obligation with respect to characterizing approaches or propelling the movement business endeavours (organization and thing). Conjecture of the movement business can use to examine the activity of the movement business on the local organizations and family unit welfare. This examination can drive the law-making body to and private money related pro to have a reasonable chart of the movement business endeavours and can provoke the gainful assignment of benefits. Contemplating on the travel industry request research uncovers the wide range of variables are viable in the travel industry, for example, the relative conversion scale, costs, pay and wellbeing. These parameters, just as different variables that do not refer to above have been utilized in many interest explores (Crouch, 1995; Johnson and Ashworth, 1990; Uysal, 1998). Numerous specialists have attempted to figure of these parameters and utilized this plan for expectation of the travel industry request. The log - direct capacity is one of the capacities that utilized generally for fitting to the travel industry demand (Crouch, 1995; Johnson and Ashworth, 1990). This sort of definition is utilized for study and inspected the reactions of various parameters on the travel industry request, in spite of the fact that this plan ought to be aligned with neighborhood information for every nation. As of late, long the traditional relapse and definition, fake neural system are exhibited as a dependable strategy for forecast of the travel industry request.

\section{Material Method}

\section{Selecting the Effective Parameters on the Tourism Demand}

A fundamental objection is that ANNs do not sufficiently reflect neuronal function. Backpropagation is a critical step, although no such mechanism exists in biological neural networks. How information is coded by real neurons is not known. Sensor neurons fire action potentials more frequently with sensor activation and muscle cells pull more strongly when their associated motor neurons receive action potentials more frequently.

As previously notice, the travel industry request is a component of the various variables. In late investigation is attempted to restrict these components for the Iraq condition. Factors, for example, conversion scale, relative value rate, sum that cost in promotions, and political issue and wellbeing are most significant parameters that affected in the travel industry request.

The original goal of the ANN approach was to solve problems in the same way that a human brain would. However, over time, attention moved to performing specific tasks, leading to deviations from biology. ANNs have been used on a variety of tasks, including computer vision, speech recognition, machine translation, social network filtering, playing board and video games, medical diagnosis and even in activities that have traditionally been considered as reserved to humans, like painting. 
Tragically, no sources found in the measure of the cost that spend on notice, consequently the impact of this parameter is disregarded in this investigation. What's more, same issue is found in parameters of the political and wellbeing. To overcome with this problem these two factors are combined together that called it "political safety". Fussy description is selected for this parameter. This parameter is set rang of the 0 and 1. For most of the years, this parameter is set as one, for year of the 2006 (changing regime in Iraq is set as 0.5), four years between the 1980-1988 (Iraq and Iran war), it is set as the zero. And it set at 0.75 for 2009.

In this study for both methods Iraq currency exchange, political safety, price rate are selected as inputs and tourism demand are set as output of the network.

\section{Artificial Neural Networks}

A fundamental objection is that ANNs do not sufficiently reflect neuronal function. Backpropagation is a critical step, although no such mechanism exists in biological neural networks. How information is coded by real neurons is not known. Sensor neurons fire action potentials more frequently with sensor activation and muscle cells pull more strongly when their associated motor neurons receive action potentials more frequently. Other than the case of relaying information from a sensor neuron to a motor neuron, almost nothing of the principles of how information is handled by biological neural networks is known.

Artificial neural networks have been used to create general relationships between variables in a given issue. Artificial neural networks are considered as a form of artificial intelligence and are somewhat capable to simulate the human brain and nervous system. As shown in Figure 1, artificial neural networks are made of a number of processing elements or nodes or neurons. The most widely used artificial neural networks in engineering purposes are known as feedforward multilayer perceptron network with back propagation algorithms with an input layer, an output layer, and one or more hidden layers (Fyfe, 2000; G.Dreyfus, n.d.; Gershenson, n.d.). Although there is no connection between the neurons in the same layer, each neuron is fully connected with the rest of neurons in the next layer (Vakili et al., 2015). The output of each node could be defined by Equation 1.

$a=f\left(\sum_{i=1}^{n} P_{i} w_{i, j}+b_{j}\right)$

Where a is the output of node $j, \mathrm{P}_{\mathrm{i}}$ is the input from $i$ th node, $\mathrm{W}_{j . i}$ is the connection weight between $j$ th node of the layer and $i$ th node of the previous layer, $\mathrm{b}_{\mathrm{j}}$ is the bias at the $j$ th node, and $f$ is the transfer function. 


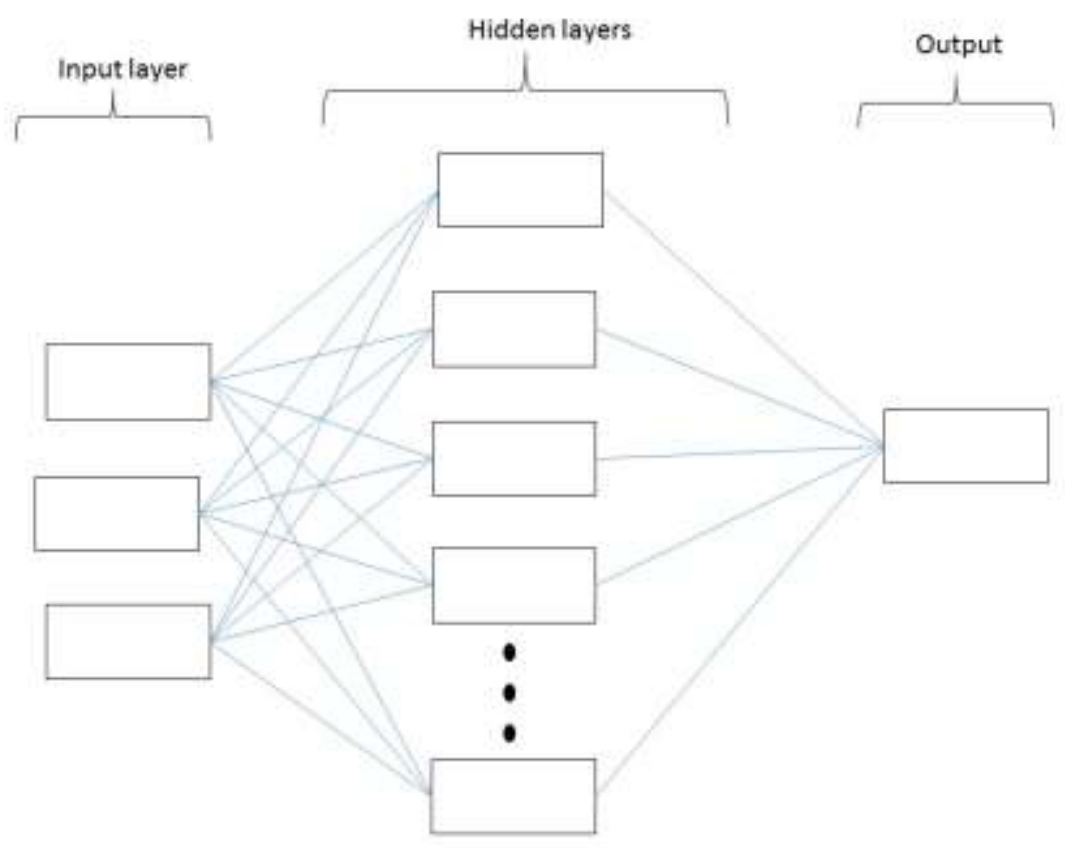

Figure 1. Schematic structure of the ANN

Sigmoid capacities are typically utilized as move capacity of every hub, in any case, different capacities, for example, hyperbolic digression capacities and straight capacities could likewise be utilized, move capacity has direct impact on the presentation of the ANN. Before choosing the exchange, work great information about the nature and conduct of the factors is vital.

The number of nodes in the input and the output layer, respectively represent the number of input and output variables. The neural network models are normally developed by dividing the available datasets into 3 subsets for training, validation, and test. In this paper ANN is initially trained by using $80 \%$ of the data while and $10 \%$ of the data is utilized in the validation process of ANN, this process has occurred during the training process to minimize over fitting ANN. The performance of the trained model is tested by the remaining $10 \%$ of the data. Thus, the training data are used for constructing the model and the validation and testing data are used for controlling the accuracy of the developed model. The difference between an experimental value (target) and the network prediction value is defined as a system error.

Where the function $\Phi \mathrm{i}(\mathrm{x})$ is called the feature that is nonlinearly mapped from the input space to the output space. This equation is nonlinear regression model because the resulting hyper-surface is a nonlinear surface hanging over the $\mathrm{m}$ dimension input space. This equation can change into a linear regression model when the input vectors $\mathrm{x}$ are mapped into vectors $\phi(\mathrm{x})$ of a high dimensional kernelinduced feature space. The nonlinear function is learned by a linear learning machine where the learning algorithm minimizes a convex function. The convex functional is expressed as the following regularized risk function, and support vector weight(w) and bias (b) can calculate by minimizing the risk function as follow (Na, Kim, Lim, \& Kang, 2008). 


$$
\begin{aligned}
& R(w)=\frac{1}{2} w^{T} w+\lambda \sum_{I=1}^{N}\left|y_{i}-f(x)_{i}\right|_{e} \\
& \left|y_{i}-f(x)_{i}\right|_{e}=\left\{\begin{array}{cr}
0 & \text { if }\left|y_{i}-f(x)_{i}\right|<\varepsilon \\
\left|y_{i}-f(x)_{i}\right|-\varepsilon & \text { otherwise }
\end{array}\right.
\end{aligned}
$$

$\lambda$ is regularization parameter, which determines the tradeoff between the approximation error and the weight vector norm. An increasing the constant $\lambda$ penalizes larger errors which decreased the approximation error also this can be happened when the weight vector norm is increased. Increasing the weight vector norm does not always lead to better generalization of the SVR model. The constants $\lambda$ and $\varepsilon$ are user-defined parameters and $\left|y_{i}-f(x)_{i}\right|_{\varepsilon}$ is called the $\varepsilon$-insensitive loss function. The loss equals zero if the prediction $\mathrm{f}(\mathrm{x})$ is within an error level $\varepsilon$, and for all other prediction points bigger than the error level $\varepsilon$, the loss is equal to the value of the difference between the prediction and the error level $\varepsilon$ (Fig. 4). Increasing the insensitivity zone (error level $\varepsilon$ ) can reduce the accuracy of the prediction, however, this increase can lead to smooth effects on modelling of the data sets that include the high level of noise (Na et al., 2008; Singh \& Gupta, 2010; Vapnik et al., 1997).

\section{Result}

We have dissected the travel industry request arrangement by considering the specific potential examples. The motivation behind breaking down the time arrangement is to present the dynamic connections among the travel industry request arrangement and improve the precision of estimates by separating the extra data accessible from the related information arrangement within reach in the tourism demand forecasting.

The goal of the paper was tourism demand forecasting execution of various fake neural system models, reaching out to visitor request determining the aftereffects of past research. Utilizing these three distinct arrangements of models, we got estimates for the quantity of voyagers from all guest markets to Iraq.

For forecast of the vacationer request two distinctive AI strategy are utilized in this paper. In the multilayer perceptron ANN various hubs number inspected in the concealed layer (from 1-10) and in each attempt the estimation of the R2 was recorded and looked at. The most extreme estimation of the R2 was seen in the neural system with the 10 hubs in the concealed layer. The pattern of the $\mathrm{R} 2$ changing is appeared in the figure 2 . The prepared connected with the 10 hubs is chosen for further examination. In the figure 2 the connection between the genuine the travel industry request and neural system expectation is appeared in the figure 2 . As clear in the figure 2 the forecast in the sum not exactly the 1 milion has great agreement with actual tourism demand. The error of prediction for both of the models were shown in the figure 3 . The fluctuation of the multi-layer perceptron is more than the SVR error prediction. 


\section{Figure 2. Amount of the $\mathbf{R}^{2}$ for different number of the node in the hidden layer}

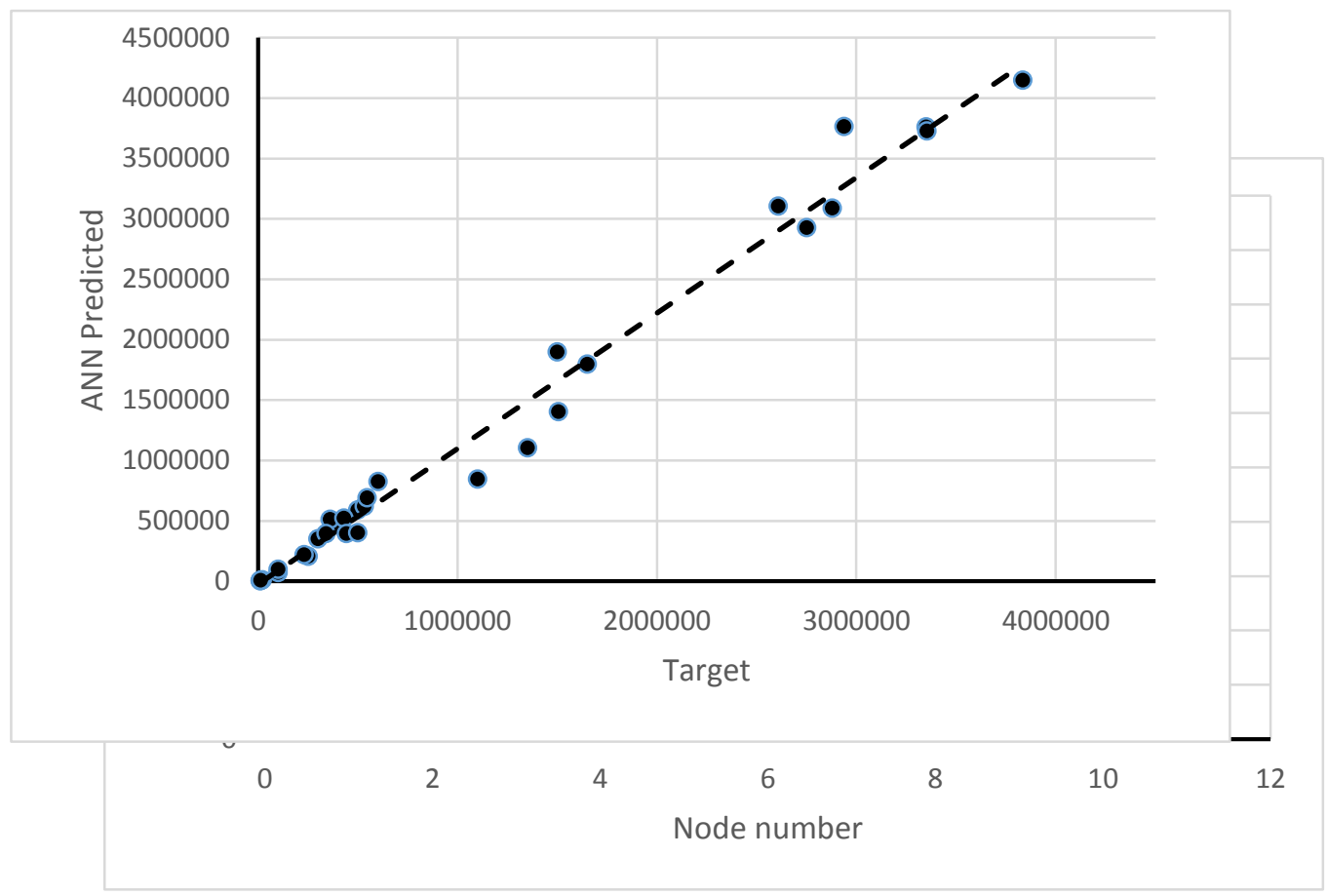

Figure 3. Comparing the prediction of the ANN with actual tourism demand

\section{References}

Boser, B., Guyon, I., \& Vapnik, V. (1992). A training algorithm for optimal margin classifiers. Proceedings of the Fifth Annual Workshop on Computational Learning Theory.

Crouch, G. I. (1995). A meta-analysis of tourism demand. Annals of Tourism Research, 22(1), 103-118. http://doi.org/10.1016/0160-7383(94)00054-V

Fyfe, C. (2000). Artificial Neural Networks and Information Theory (1.2 ed.).

G.Dreyfus. (n.d.). Neural Networks Methodology and Applications. Springer-Verlag Berlin Heidelberg 2005.

Gershenson, C. (n.d.). Artificial Neural Networks for Beginners, 1-8.

Johnson, P., \& Ashworth, J. (1990). Modelling tourism demand: A summary review. Leisure Studies. Retrieved from http://www.tandfonline.com/doi/pdf/10.1080/02614369000390131 
Kecman, V. (2001). Learning and soft computing: support vector machines, neural networks, and fuzzy logic models. MIT press.

Kon, S., \& Turner, L. (2005). Neural network forecasting of tourism demand. Tourism Economics. Retrieved from http://www.ingentaconnect.com/content/ip/tec/2005/00000011/00000003/art00001

Law, R. (2001). The impact of the Asian financial crisis on Japanese demand for travel to Hong Kong: A study of various forecasting techniques. Journal of Travel \& Tourism Marketing. Retrieved from http://www.tandfonline.com/doi/abs/10.1080/10548400109511558

Lim, C. (1997). Review of international tourism demand models. Annals of Tourism Research, 24(4), 835849. http://doi.org/10.1016/S0160-7383(97)00049-2

Na, M. G., Kim, J. W., Lim, D. H., \& Kang, Y. J. (2008). Residual stress prediction of dissimilar metals welding at NPPs using support vector regression. Nuclear Engineering and Design, 238, 1503-1510.

Schölkopf, B., \& Burges, C. (1999). Advances in kernel methods: support vector learning. MIT press.

Singh, S. K., \& Gupta, A. K. (2010). Application of support vector regression in predicting thickness strains in hydro-mechanical deep drawing and comparison with ANN and FEM. CIRP Journal of Manufacturing Science and Technology, 3(1), 66-72.

Song, H., \& Li, G. (2008). Tourism demand modelling and forecasting-A review of recent research. Tourism Management, 29(2), 203-220. http://doi.org/10.1016/j.tourman.2007.07.016

Vakili, A. H., Davoodi, S., Arab, A., Researcher, Y., Club, E., Branch, E., ... Tebal, N. (2015). Use of Artificial Neural Network in Predicting Permeability of Dispersive Clay Treated With Lime and Pozzolan. Ijsres, 3(1), 23-37.

Vapnik, V., Golowich, S. E., \& Smola, A. (1997). Support vector method for function approximation, regression estimation, and signal processing, 281-287.

Witt, S. F., \& Witt, C. A. (1995). Forecasting tourism demand: A review of empirical research. International Journal of Forecasting, 11(3), 447-475. http://doi.org/10.1016/0169-2070(95)00591-7

\section{Copyrights}

Copyright for this article is retained by the author(s), with first publication rights granted to the journal.

This is an open-access article distributed under the terms and conditions of the Creative Commons Attribution license (http://creativecommons.org/licenses/by/4.0/). 